\title{
ESTUDIO DE COBERTURA DE RADIOFRECUENCIA Y POTENCIA PARA EL REDISEÑO DEL SISTEMA DE TRANSMISIÓN: UN CASO ESTUDIO EN LA EMISORA MARINA STEREO 90.7 FM EN LA CIUDAD DE BARRANQUILLA

\author{
RADIOFREQUENCY COVERAGE AND POWER STUDY FOR THE REDESIGN \\ OF THE TRANSMISSION SYSTEM: A CASE STUDY IN THE MARINE \\ STEREO 90.7 FM STATION IN BARRANQUILLA CITY
}

\section{Ing. Ronald Ariza Hurtado*, José Cuello Navarro*, Carlos Barraza Peña*, MSc. Kelvin Beleño Sáenz *, MEng. Evert de los Ríos Trujillo *, MSc. José Escorcia- Gutierrez ${ }^{*}{ }^{* *}$}

* Universidad Autónoma de Caribe, Facultad Ingeniería, Grupo de Investigación IETUAC.

Calle 90 \# 46 - 112, Barranquilla, Atlántico, Colombia.

E-mail: \{ronald.ariza, carlos.barraza1, jose.cuello1, kelvin.beleno, evert.delosrios23, jose.escorcia47\}@uac.edu.co

*** Escuela Naval de Suboficiales A.R.C. "Barranquilla", Centro de Investigaciones, Grupo de Investigación GITIN.

Calle 58 Esquina Vía 40, Barranquilla, Atlántico, Colombia.

E-mail: jose.escorcia23@gmail.com

Resumen: El presente artículo describe el estudio e implementación de una mejora realizada a la estación de radio Marina Stereo 90,7 FM, la cual presenta fallas en su potencia de operación del sistema de transmisión, generando baja cobertura en la potencia de recepción, por tal motivo el objetivo de la mejora implementada es el rediseño del sistema de transmisión por medio de herramientas de diagnóstico, medición y validación de datos en campo, análisis de estos y toma de decisiones para determinar las innovaciones necesarias en materia de infraestructura para hacer más optima la señal transmitida y por consecuente, hacer que el sistema de radio en materia de cobertura en potencia tenga mayor alcance y no se vea afectado por factores como irregularidades del terreno, hacia el cual se proyecta la señal, desarrollo urbanístico de edificios y vivienda vertical.

Palabras clave: Potencia, señal, ondas de radio, transmisión, antena.

\begin{abstract}
The present article describes the study and implementation of an improvement made to the radio station Marina Stereo 90.7 FM, which presents failures in its operation power of the transmission system, which generates a low coverage in received power, for that reason the objective of the implemented improvement is the redesign of this transmission system by means of diagnostic tools, measurement and validation of data in field, analysis of these and decision making based on them in order to determine the necessary innovations in terms of infrastructure required to make the transmitted signal more optimal and consequently, make the radio system in terms of power coverage has greater range and is not affected by factors such as irregularities of the terrain, to which the signal is projected, urban development of buildings and vertical housing.
\end{abstract}

Keywords: Power, signal, radio waves, transmission, antenna. 


\section{INTRODUCCIÓN}

La radio, en la historia de la comunicación tiene su lugar como una innovación tecnológica que marcó un antes y un después en el mundo. Desde sus inicios hasta la actualidad, la radio es tenida en cuenta como un medio de comunicación masiva, ya que, gracias a sus avances predecesores como el descubrimiento de las ondas electromagnéticas, la electricidad, el electromagnetismo y el desarrollo del telégrafo, esta permite que muchas personas se eduquen, se informen de los acontecimientos del día a día, se diviertan y hasta conozcan de la cultura de otras localidades.

Actualmente en Colombia, el servicio público gratuito de la radio ha tenido un crecimiento considerable desde hace unas décadas. Según la subdirección de radiodifusión sonora del Ministerio de Tecnologías de la Información y las Comunicaciones (MinTIC) [1], la radio es el medio de comunicación con mayor alcance y difusión en Colombia, esto lo reflejan las estadísticas que indican que el $99,1 \%$ de la población nacional (48,3 millones de habitantes aprox.) tiene acceso a este medio. Adicional a esto, hoy por hoy 1.596 emisoras se encuentran operando y haciendo uso del espectro radioeléctrico asignado por la entidad reguladora (ANE, Agencia nacional del espectro) en las bandas de AM (Amplitud modulada) y FM (frecuencia modulada). De estas dos bandas 1243 son FM y 353 son AM.

Ahora bien, todas esas emisoras ubicadas en las diferentes bandas asignadas deben garantizar una correcta transmisión, proyección y difusión de la señal que están emitiendo, esto con el objetivo de abarcar la mayor área territorial posible y así, lograr llegar a más habitantes que deseen escuchar de la emisora. Ampliar la zona cobertura dentro de una zona geográfica es para el sistema de transmisión una de las metras principales cuando se implementa una emisora de radiodifusión sonora. Para el caso de las emisoras que operan en forma comercial, en FM; esto adquiere un valor superlativo en función de la cobertura que tendrá su emisión, ya que de ella dependerá la cantidad de oyentes potenciales. Tener mayor alcance con una emisora significa además una oferta muy tentadora para que los auspiciantes coloquen su publicidad en estaciones de radio. Más habitantes que deseen escuchar de la emisora. Es por tal motivo que en el presente documento se plantea el rediseño del sistema de transmisión de la emisora Marina Stereo 90.7 FM, basado en la problemática de baja

Universidad de Pamplona

I. I. D. T. A. cobertura en potencia sobre la localidad norte centro histórico de la ciudad de Barranquilla teniendo en cuenta que esta (la potencia de transmisión) es uno de los parámetros técnicos esenciales descritos en el Plan Técnico Nacional de Radio Difusión Sonora (PTNRS) en Frecuencia Modulada (FM) [2], por lo tanto, se realizaron actividades de medición en campo, recolección y validación de los datos obtenidos en dichas mediciones frente a los establecidos por el MinTIC. Posterior a esto, a dichos valores se les realizó un proceso de emulación en la herramienta computacional Matlab, describiendo de forma gráfica el comportamiento radioeléctrico de la señal de potencia en el transmisor de la emisora tanto en los instantes de antes y después de la implementación de la mejora, que a continuación se va a explicar, con el objetivo de generar una proyección o tendencia pronóstico de la cobertura en potencia.

\section{ESTADO DEL ARTE}

En el ámbito de la radiodifusión es muy beneficioso conocer el alcance que se tiene con las ondas de radio transmitidas, debido a que al realizar este tipo de estudios es posible identificar si existen zonas en las que sea difícil la sintonización de las estaciones de radio, y, con base a ello, contemplar la posibilidad de implementar cambios y mejoras en el sistema de transmisión para lograr dar solución a la problemática en mención.

A partir del escenario descrito se llegan a elaborar numerosas investigaciones con la finalidad de analizar el fenómeno de radiodifusión en el área de interés, llegando a determinar los factores que más repercuten en la calidad de la señal emitida. Un ejemplo de ello es el trabajo redactado por Molina C. [3] quien describe características básicas de las señales análogas, que son las generadas por la radiodifusión en la actualidad, y diversos factores que pueden afectar la transmisión de este tipo de señales. De manera similar, Calvopiña Hinojosa [4] presenta un estudio de los aspectos que inciden en el ancho de banda y el nivel de intensidad de campo eléctrico de la señal de radio a transmitir, incluyendo las características técnicas de los equipos e instrumentos utilizados por las emisoras de FM; además, propone una serie de ideas que generarían una mejora en la calidad de dicha señal.

Para el desarrollo del proyecto descrito en el presente artículo se consultaron referencias como 
los textos redactados por Gallardo, S. [5] y Henze, A. [6], en donde el primer autor detalla los elementos principales que componen un sistema de telecomunicaciones, mientras que el segundo denota las variables de potencia presentes en la transmisión y los principios que rigen a los dispositivos usados para las mediciones correspondientes. En adición, se tuvo en cuenta la normatividad del Plan Técnico Nacional de Radiodifusión Sonora (PTNRS) de Frecuencia Modulada estipulada por el MinTIC, esto con la finalidad de garantizar que las modificaciones a realizar en el sistema de transmisión puedan ser implementadas sin desacatar la reglamentación en ningún aspecto.

En la misma línea de desarrollo de la presente propuesta de ingeniería, se destaca el estudio de rediodifusión de la emisora Libre 93.9 FM en la ciudad de Babahoyo, Ecuador, por parte de Ruíz S. [7], en el cual se buscó identificar los requerimientos técnicos necesarios para la actualización de la red de transmisión de la emisora para mejorarla y ajustarla a la actualización de la Norma Técnica para el Servicio de Radiodifusión Sonora, garantizando el adecuado funcionamiento de la estación de radio.

Por último, un gran aporte para llevar a cabo este proyecto fue el estudio realizado por Mejía, M., \& Pacheco, L [8] previamente en la estación de la emisora de Marina Stereo 90.7 FM en Barranquilla. En este, se desarrolla una serie de mediciones de los niveles de potencia de la señal generada por la emisora en diferentes puntos de la ciudad con la finalidad determinar la calidad que presenta la estación en diferentes zonas. En base a esta información, y haciendo una comparación respecto a la calidad de otras emisoras, se logra determinar el problema de cobertura que presenta Marina Stereo, evidenciando lo significativo que es la elaboración de la propuesta del rediseño descrito a continuación.

\section{PROPUESTA DE INGENIERÍA}

4.

Nota: Para la propuesta de ingeniería y los resultados del estudio e implementación de dicha propuesta solo se mostrarán algunos materiales utilizados, debido a que está prohibido la exhibición o diagramación al público en general de las condiciones técnicas internas de la empresa militar, así mismo instalaciones o áreas internas por razones de la seguridad nacional de las Fuerzas Militares de Colombia.

Universidad de Pamplona

I. I. D. T. A.
Para la realización de la propuesta de ingeniería se estudió una zona delimitada de la ciudad de Barranquilla, Atlántico bajo la toma de 24 muestras en campo o puntos, 12 principales y 12 de respaldo (todos aleatorios). Estas muestras, las cuales se harán en diferentes horarios de mañana, tarde y noche (según la normatividad expuesta por la entidad reguladora encargada del espectro en Colombia, ANE), para así lograr apreciar en forma numérica y gráfica el estado de cobertura en potencia actual de la emisora Marina Stereo sobre la localidad norte centro histórico de la ciudad comparándola frente a emisoras vecinas que en este caso corresponden a las emisoras de Caracol Radio 90.1 FM y Olímpica Stereo 92.1 FM.

Posterior a la recolección de las muestras de los niveles de cobertura en potencia de las tres emisoras mencionadas anteriormente en una zona geográfica definida de la ciudad de Barranquilla, se procede a realizar el análisis de datos por medio de la herramienta computacional Statgraphics. De este análisis se espera que junto con la simulación del fenómeno estudiado mediante Matlab se represente a través de diagramas de cajas y bigotes la comparación de los niveles de cobertura en potencia de la emisora Marina Stereo con sus emisoras vecinas para así determinar las mejoras necesarias que se le deben hacer al sistema de transmisión a nivel de hardware para lograr una optimización e incremento del nivel de potencia en la señal de radiodifusión sonora que se está emitiendo. Esta toma de decisión de las mejoras que se van a poner en marcha va a ser mucho más eficaz gracias al mapa de calor que se obtiene de Matlab por medio del paquete Antenna Toolbox, el cual nos proporciona funciones para el diseño, análisis y visualización de propagación de antenas y sus coberturas, además que nos es posible diseñar antenas independientes y crear arreglos de antenas mediante elementos predefinidos con geometría parametrizada. Dentro de los comandos de propagación RF se puede visualizar y emular la cobertura y los enlaces de comunicación en un mapa de terreno 3D.

Gracias a esta herramienta que da como resultado un mapa de calor se evaluará los emplazamientos de antenas en cuanto al punto de la transmisión y los puntos receptores, trazando así enlaces de comunicación y visualización de la cobertura y potencia de la señal.

Posterior a todo el estudio de las cifras obtenidas (que se muestran al detalle en la sección de Resultados) se originaron una serie de propuestas 
de mejora y cambios que van a lograr darle solución al problema descrito en un principio. Estas propuestas o planes de mejora son: 1 . Rediseñar el sistema de transmisión teniendo en cuenta los parámetros técnicos esenciales del Plan Técnico Nacional de Radiodifusión Sonora (PTNRS); 2. Proponer el incremento de la potencia del transmisor de FM de 400W a 500W; 3. Cambio de la estructura que soporta a la antena emisora a nivel de obra civil; y 4. Evaluación de estructura tipo torre metálica con soporte para peso y anclaje de fuerte viento.

Se propone ubicar la antena a una mayor altura en la torre con respecto al suelo, ya que, así como se aprecia en la Tabla 1, esta modificación genera cambios significativos que contribuyen a una mejora del sistema.

Tabla 1. Estimación de la variación del alcance de la señal ante el aumento de la altura de la antena.

Fuente: Angeletti.com (2020), Alcance estimado de las Emisoras de FM y TV abierta https://ingangeletti.com/alcance-estimado-de-lasemisoras-de-fm-y-tv-abiertal

\begin{tabular}{|c|c|}
\hline Altura de la Antena & Alcance Óptico \\
\hline 10 metros & $18 \mathrm{Km}$ \\
\hline 15 metros & $21 \mathrm{Km}$ \\
\hline 20 metros & $23 \mathrm{Km}$ \\
\hline 25 metros & $25 \mathrm{Km}$ \\
\hline 30 metros & $27 \mathrm{Km}$ \\
\hline 40 metros & $31 \mathrm{Km}$ \\
\hline 45 metros & $32 \mathrm{Km}$ \\
\hline 50 metros & $34 \mathrm{Km}$ \\
\hline 60 metros & $37 \mathrm{Km}$ \\
\hline 70 metros & $39 \mathrm{Km}$ \\
\hline
\end{tabular}

Para este caso se plantea el montaje de la antena a una altura de 45 metros, lo cual nos brinda una distancia de alcance de la señal de aproximadamente $32 \mathrm{~km}$. Se escoge dicha altura porque se debe tener en cuenta que la geografía de la ciudad no es completamente plana por lo que pese a las alteraciones del terreno y a las edificaciones de construcción vertical esta altura proporcionará una distancia de emisión suficiente para la señal de radiofrecuencia.

También se plantea el cambio del cableado coaxial (cable que lleva la señal del transmisor a la antena) y conectores nuevos de cobre junto con la aplicación de la técnica de enfasamiento de 4 antenas dipolo de polarización circular OMB SGP diseñada para mantener una potencia continua de $3 \mathrm{~kW}$ a $5 \mathrm{~kW}$ por cada elemento y su constitución en acero inoxidable garantiza la inalterabilidad de sus especificaciones. Es una antena de banda ancha recomendada para condiciones atmosféricas extremas. Adicionalmente, brinda soporte para irradiar señal de radiodifusión por medio de transmisores que usen la tecnología digital DBA FM (Digital Audio Broadcasting) convirtiéndola en una antena que estará a la vanguardia en tecnología de radiodifusión sonora por muchos años.

Ahora bien, la técnica mencionada anteriormente que se conoce como enfasamiento, es aquella que consiste en agrupar antenas idénticas conectadas al mismo equipo de radio con el objetivo de conseguir una mayor ganancia y directividad del sistema de antenas con respecto a una única antena ("Enfasamientos", 2018). Esta configuración se propone implementar en el sistema solución, así como se ilustra en la Figura 1.

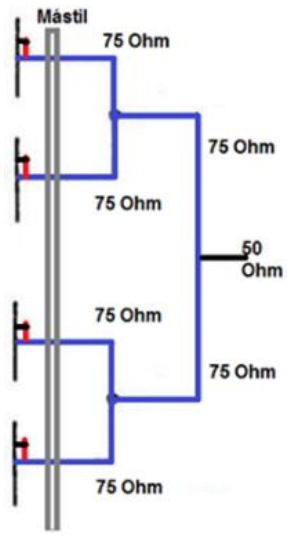

Figura 1. Enfasamiento de 4 antenas dipolo con sus respectivas impedancias equivalentes.

Fuente:https://sites.google.com/site/ea7ahg/antena lenfasamientos

Para el caso de la estructura que soporta al conjunto de antenas, se propone cambiar la estructura poste de cemento por una torre metálica venteada con una altura máxima de 45 metros, que, según la Tabla 1, deberá ser suficiente para ofrecer cobertura en la zona de estudio.

Este tipo de torre se caracteriza por brindar rigidez contra el suelo, soporta peso, vientos extremos y se encuentra galvanizada lo cual indica un mayor soporte para las variaciones de clima, el salitre y la humedad. Además, cumple con la normal de señalización de la OACI (Organización de aviación civil internacional) con sus colores rojo-blancorojo colocados en la misma proporción. Lleva también en la parte superior el balizaje nocturno de luces color rojo, su sistema de puesta a tierra y el debido pararrayos. (Para más información visitar: Ingeniería \& Soporte, (2016). Catálogo de torres. Disponible en: https://bit.ly/36qQAoV) 
Adicionalmente, como parte de la propuesta de ingeniería se sugiere la reorientación de las antenas con un nuevo azimut hacia el barrio los nogales el cual es el punto de mayor altura dentro del área de estudio.

Por último, en base a la Tabla 2, el tipo de línea de transmisión y conector que se sugiere son el cable coaxial Cell Flex $7 / 8$ " con impedancia de $50 \Omega$ con bajo porcentaje de perdidas por distancia ( $5 \%$ de la potencia en $30 \mathrm{mts}$ ), viene aprovisionado de conectores para el acoplamiento ideal con antenas OMB SGP WB lo cual se traduce a una máxima transferencia de potencia entre el transmisor y la entrada de la antena.

Tabla 2. Pérdidas de Potencia presente en diferentes tipos de cable.

Fuente: Angeletti.com (2020), Alcance estimado de las Emisoras de FM y TV abierta https://ingangeletti.com/alcance-estimado-de-lasemisoras-de-fm-y-tv-abiertal

\begin{tabular}{|c|c|}
\hline Tipo de Cable & Pérdida Estimada \\
\hline Coaxial RG 213 & $\begin{array}{c}30 \% \text { de la Potencia a } \\
30 \text { m de Distancia }\end{array}$ \\
\hline RG 11 & $\begin{array}{c}20 \% \text { de la Potencia a } \\
30 \text { m de Distancia }\end{array}$ \\
\hline Cell Flex 1/2, & $\begin{array}{c}10 \% \text { de la Potencia a } \\
30 \text { m de Distancia }\end{array}$ \\
\hline Cell Flex 7/8, & $\begin{array}{c}5 \% \text { de la Potencia a 30 } \\
\text { m de Distancia }\end{array}$ \\
\hline
\end{tabular}

\section{RESULTADOS}

Los puntos escogidos para la toma de muestras para la validación de los datos y posterior generación de los mapas de calor se muestran en la Figura 2. Los datos obtenidos fueron captados a través del instrumento de medición de potencia en decibeles, Analizador de espectro (BK Precisión 2650a). Con este instrumento y haciendo uso de la normatividad expuesta por el Plan Técnico Nacional de Radiodifusión Sonora (PTNRS) en frecuencia modulada (FM), se logró establecer 3 rangos definidos para calificar la calidad audible en la recepción consolidados en la Tabla 3.

En la Tabla 4 se detalla la descripción técnica por punto, cobertura y nivel de potencia de las muestras seleccionadas para el estudio.

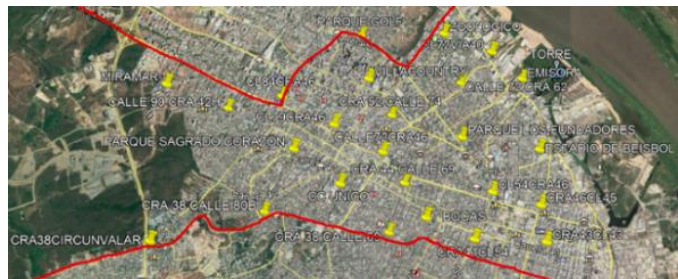

Figura 2. Puntos (aleatorios en el área afectada) en los que se hicieron las mediciones de cobertura en potencia.

Fuente: Google Earth Pro

Tabla 3. Rangos para clasificación de la calidad audible de una señal recepcionada.

Fuente: Elaboración Propia.

\begin{tabular}{|c|c|}
\hline CALIDAD SEÑAL & RANGO \\
\hline Baja & $-75 \mathrm{dBm}$ a $-\infty$ \\
\hline Media & $-70 \mathrm{dBm}$ a $-74 \mathrm{dBm}$ \\
\hline Alta & $-69 \mathrm{dBm}$ y mayores. \\
\hline
\end{tabular}

Tabla 4. Puntos en los que se realizaron las mediciones de la calidad y nivel de potencia.

Fuente: Elaboración Propia.

\begin{tabular}{|c|c|c|}
\hline Puntos & Cobertura & $\begin{array}{c}\text { Nivel de } \\
\text { potencia } \\
\text { (dBm) }\end{array}$ \\
\hline $\begin{array}{c}\text { CRA 38 - } \\
\text { Circunvalar }\end{array}$ & Calidad Baja & -77.0 \\
\hline C.C Único & Calidad Media & -74.1 \\
\hline $\begin{array}{c}\text { Parque Sagrado } \\
\text { Corazón }\end{array}$ & Calidad Media & -75.2 \\
\hline Zoológico & Calidad Alta & -56.2 \\
\hline CRA 46 - 72 & Calidad Alta & -68.6 \\
\hline Calle 54 CRA 46 & Calidad Alta & -59.9 \\
\hline Calle 46 Calle 45 & Calidad Alta & -61.7 \\
\hline Villa Country & Calidad Alta & -69.8 \\
\hline Miramar & Calidad Baja & -74.1 \\
\hline CRA 46 Calle 84 & Calidad Media & -72.8 \\
\hline Parque Los & Calidad Alta & -53.4 \\
\hline Fundadores & Calidad Alta & -47.5 \\
\hline Calle 72 vía 40 & Calidad Media & -54.8 \\
\hline CRA 38 Calle 69 & Calidad Alta & -69.5 \\
\hline CRA 41 Calle 54 & Calida \\
\hline CRA 43 Calle 43 & Calidad Alta & -50.8 \\
\hline Calle 72 CRA 62 & Calidad Media & -50.8 \\
\hline Estadio de Béisbol & Calidad Alta & -53.3 \\
\hline Calle 90 CRA42H & Calidad Baja & -79.1 \\
\hline 7 Bocas & Calidad Media & -72.3 \\
\hline Calle 79 CRA 46 & Calidad Media & -64.0 \\
\hline CRA 38 Calle 80 & Calidad Baja & -77.3 \\
\hline Parque Golf & Calidad Media & -61.3 \\
\hline CRA 44 Calle 69 & Calidad Media & -64.0 \\
\hline CRA 52 Calle 74 & Calidad Alta & -62.6 \\
\hline
\end{tabular}


Los datos consolidados en la Tabla 4 se pueden observar de forma gráfica en la Figura 3 gracias a la emulación que se hizo con la herramienta computacional Matlab y su paquete Antenna Toolbox que arroja como resultado un mapa de calor que ilustra el comportamiento de cómo es la propagación de la señal de la emisora Marina Stereo 90.7 FM en el área urbana teniendo como referencia la antena emisora (punto localizador rojo).

En el área se observa que la señal emitida se le considera de calidad alta es muy pobre en extensión, se observa que debido a variaciones en el terreno o por obstáculos como las edificaciones $\mathrm{y}$ viviendas verticales que hacen que la señal de la emisora Marina Stereo 90.7 FM presente una baja notoria en su alcance. También se observa que, la sección en la que la señal es considerada Baja es muy considerable, tanto así que se pueden apreciar secciones en las que la señal es prácticamente nula.

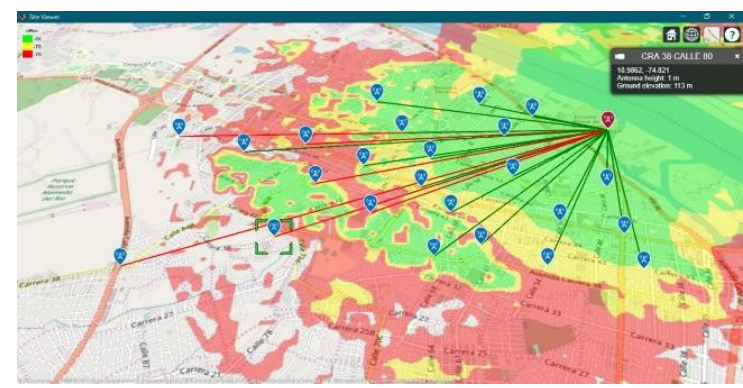

Figura 3. Mapa de Calor de la emisora Marina Stereo 90.7 FM

Fuente: Mejía, M., \& Pacheco, L. (2019). [8]

Cabe aclarar que al momento de la investigación no surgieron problemas de interferencias con otras emisoras, por tanto, la variable a analizar es únicamente la potencia, teniendo fija la frecuencia de 90.7 FM. Luego para el tratamiento y análisis de los datos de potencia de la señal recibida gracias a la herramienta computacional Statgraphics se obtuvieron los gráficos comparativos de las mediciones en campo de la emisora objeto del proyecto, frente a la emisora Caracol radio 90.1 FM (Figura 6) y Olímpica Stereo 92.1 FM (Figura 7).

Adicional a esto también se realizaron los respectivos mapas de calor de las emisoras vecinas con el objetivo de hacer mucho más gráfica la comparativa entre las tres emisoras en su estado actual y así darle un mayor soporte a la posterior obtención y análisis de resultados de la propuesta planteada. Los mapas de calor de las emisoras

Universidad de Pamplona

$$
\text { I. I. D. T. A. }
$$

vecinas (Olímpica Stereo y Caracol Radio) se observan en las Figuras 4 y 5 .

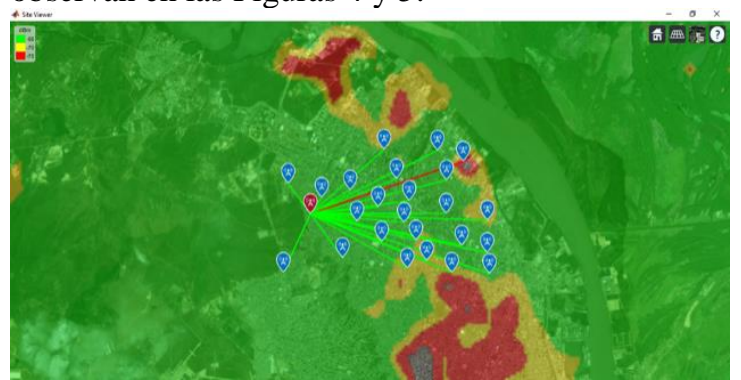

Figura 4. Mapa de Calor de la emisora Caracol Radio 90.1 FM

Fuente: Mejía, M., \& Pacheco, L. (2019). [8]

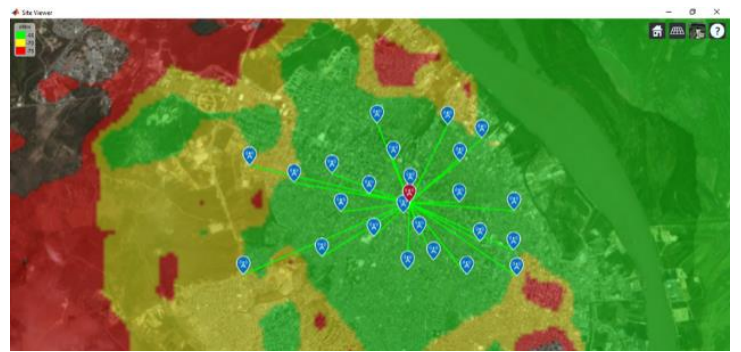

Figura 5. Mapa de Calor de la emisora Olímpica Stereo 92.1 FM

Fuente: Mejía, M., \& Pacheco, L. (2019). [8]

Con la observación de dichas gráficas se puede afirmar entonces que la emisora Marina Stereo en comparación con sus emisoras vecinas presenta una baja sustancial en aspecto de cobertura en potencia lo cual indica que es necesario realizar mejoras al sistema de transmisión para aumentar el alcance de dicha señal, y así, más oyentes puedan sintonizar la emisora sin ruidos, interferencias o distorsiones.

Las figuras 6 y 7 muestran en forma de Diagrama de Caja y bigotes la comparativa del desplazamiento notorio hacia valores bajos del nivel de potencia de la señal de la emisora Marina Stereo frente a sus emisoras vecinas.

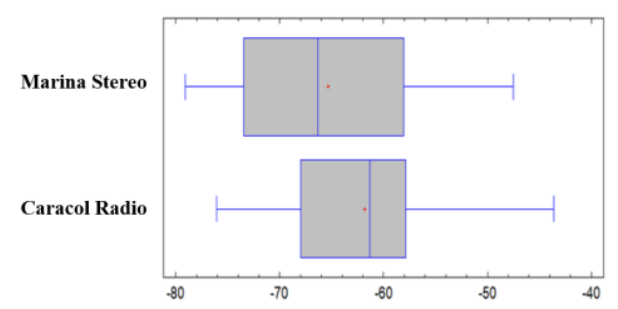

Figura 6. Diagrama de Caja y Bigotes: Análisis de datos de las mediciones en campo de la emisora Marina Stereo 90.7 FM frente a la emisora Caracol Radio 90.1 FM. 
Fuente: Mejía, M., \& Pacheco, L. (2019). [8]

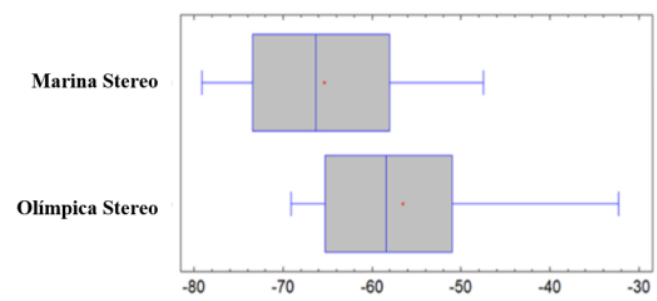

Figura 7. Diagrama de Caja y Bigotes: Análisis de datos de las mediciones en campo de la emisora Marina Stereo 90.7 FM frente a la emisora Olímpica Stereo 92.1 FM.

Fuente: Mejía, M., \& Pacheco, L. (2019). [8]

Luego de la implementación de las mejoras realizadas (explicadas en la sección de Propuesta de Ingeniería), por medio de simulaciones se realizaron nuevas mediciones pronóstico del nivel de señal esperado según los parámetros que se variaron del sistema inicial, tales como altura de la antena, tipo de cable coaxial, potencia del transmisor y orientación de la antena.

En la Figura 8 y 9 se muestra ahora el resultado de la nueva cobertura en potencia pronosticada del rediseño del sistema de transmisión frente a las emisoras vecinas.

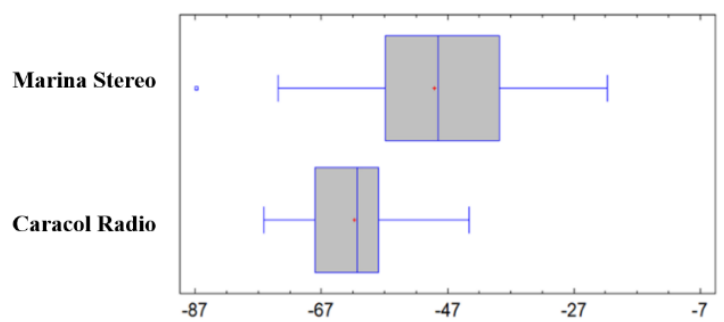

Figura 8. Diagrama de Caja y Bigotes: Evaluación de la nueva cobertura en potencia pronóstico del rediseño del sistema de transmisión frente a la emisora vecina Caracal Radio.

Fuente: Mejía, M., \& Pacheco, L. (2019) [8].

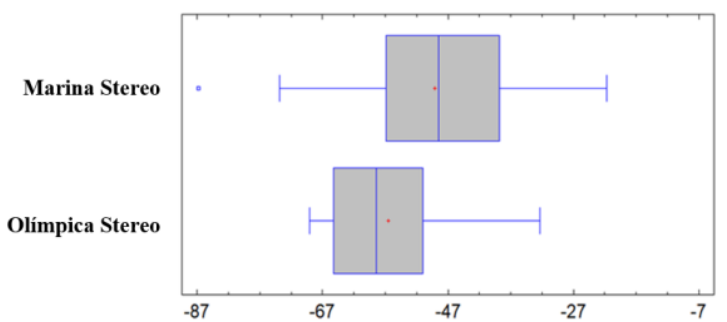

Figura 9. Diagrama de Caja y Bigotes: Evaluación de la nueva cobertura en potencia pronóstico del rediseño del sistema de transmisión frente a la emisora vecina Olímpica Stereo.

Fuente: Mejía, M., \& Pacheco, L. (2019) [8].
Tabla 5. Nueva cobertura en potencia pronóstico.

Fuente: Elaboración Propia.

\begin{tabular}{|c|c|c|}
\hline $\begin{array}{c}\text { MARINA STEREO } \\
\text { 90.7 FM }\end{array}$ & ANTES & $\begin{array}{c}\text { PRONOS } \\
\text { TICO }\end{array}$ \\
\hline Puntos & dBm & dBm \\
\hline $\begin{array}{c}\text { CRA 38 - } \\
\text { Circunvalar }\end{array}$ & -77.0 & -61 \\
\hline C.C Único & -74.1 & -53 \\
\hline $\begin{array}{c}\text { Parque Sagrado } \\
\text { Corazón }\end{array}$ & -75.2 & -57.5 \\
\hline Zoológico & -56.2 & -39.2 \\
\hline CRA 46 - 72 & -68.6 & -48.3 \\
\hline Calle 54 CRA 46 & -59.9 & -38.5 \\
\hline Calle 46 Calle 45 & -61.7 & -33.3 \\
\hline Villa Country & -69.8 & -73.9 \\
\hline Miramar & -74.1 & -86.8 \\
\hline CRA 46 Calle 84 & -72.8 & -69.6 \\
\hline Parque Los & -53.4 & -31.9 \\
\hline Fundadores & -47.5 & -21.7 \\
\hline Calle 72 vía 40 & -54.8 & -31.8 \\
\hline CRA 38 Calle 69 & -69.5 & -41.3 \\
\hline CRA 41 Calle 54 & -50.8 & -41.9 \\
\hline CRA 43 Calle 43 & -50.8 & -48.8 \\
\hline Calle 72 CRA 62 & -53.3 & -28.9 \\
\hline Estadio de Béisbol & -79.1 & -72.1 \\
\hline Calle 90 CRA 42H & -72.3 & -40.4 \\
\hline 7 Bocas & -64.0 & -55.5 \\
\hline Calle 79 CRA 46 & -77.3 & -56.3 \\
\hline CRA 38 Calle 80 & -61.3 & -50.8 \\
\hline Parque Golf & -64.0 & -44.8 \\
\hline CRA 44 Calle 69 & -62.6 & -50.5 \\
\hline CRA 52 Calle 74 & \\
\hline
\end{tabular}

En comparación con la Figura 3, el nuevo mapa de calor de la potencia pronóstico de la emisora Marina Stereo se observa en la Figura 10.

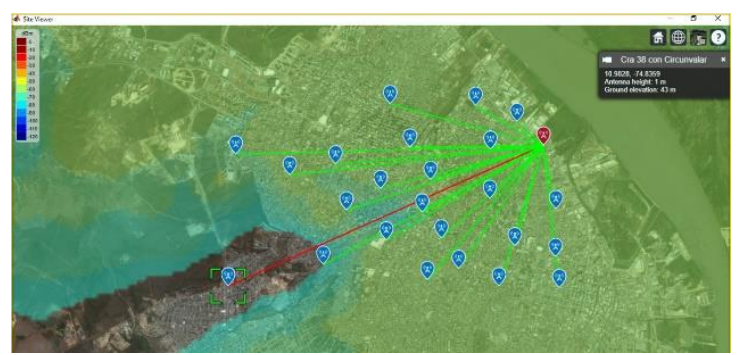

Figura 10. Mapa de calor de la potencia pronóstico de la emisora Marina Stereo.

Fuente: Mejía, M., \& Pacheco, L. (2019) [8].

Y finalmente en la Figura 11 y 12 se muestra el estado del nivel de potencia antes y después de la implementación de la mejora planteada como propuesta solución. 


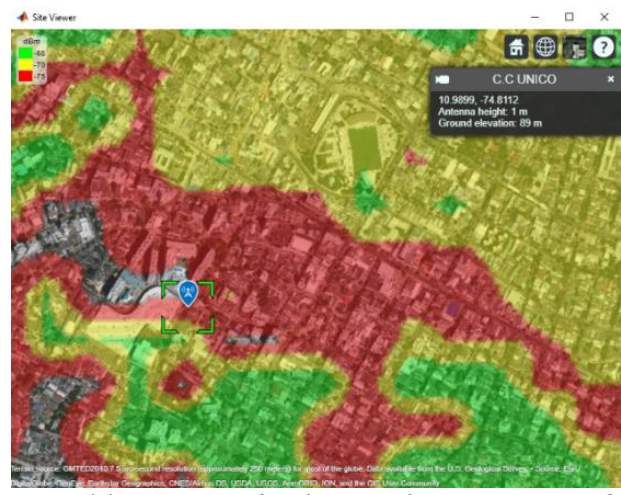

Figura 11. Antes de la implementación de la propuesta solución.

Fuente: Elaboración Propia.

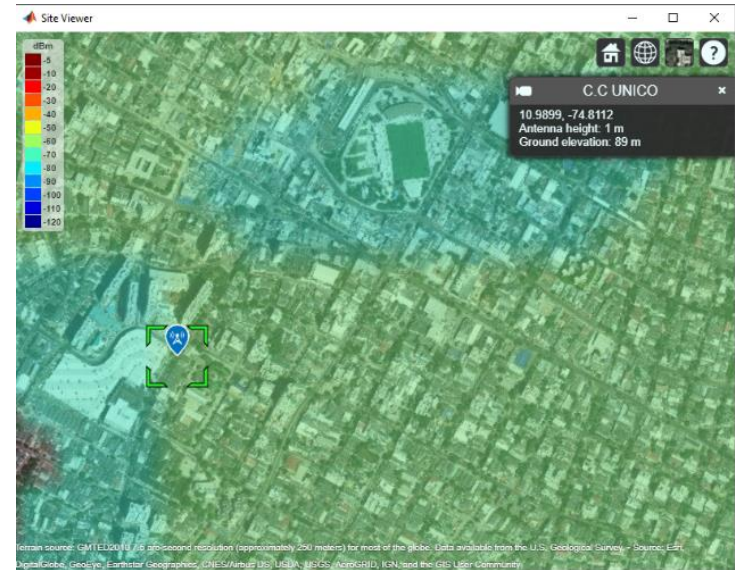

Figura 12. Después de la implementación pronóstico de la propuesta solución.

Fuente: Elaboración Propia.

\section{CONCLUSIONES}

Con el desarrollo del proyecto ya descrito se concluye que es posible incrementar la eficiencia de la transmisión de ondas de radio FM de la estación radial Marina Stereo al modificar aspectos como el aumento de la altura de las antenas con respecto al suelo, la reorientación de las antenas hacia la zona afectada, el cambio de cableado y conectores, y el incremento en potencia en el transmisor, esto con el fin de alcanzar la cobertura necesaria para recibir una señal audible de buena calidad en una mayor área de la ciudad de Barranquilla. Aunque las mejoras planteadas fueron inicialmente propuestas para dar solución a problemas en el entorno de la radiodifusión, también pueden adaptarse y ser útiles para demás tipos de servicios inalámbricos que utilizan el espectro radioeléctrico.
Por último, si bien se logró observar una mejora en gran medida la emisión de este tipo de señales análogas, la realidad es que siguen presentándose ciertos límites e inconvenientes. Este aspecto se tuvo en cuenta en la elaboración del estudio al sugerir el uso de antenas de tipo dipolo de polarización circular que soporta la tecnología digital DBA, ya que, además de brindar una solución satisfactoria a los problemas actuales, la implementación de estas antenas significa estar consciente de la era de digitalización de los sistemas de radiodifusión, en la cual será imprescindible adaptar el estándar DBA para ofrecer un mejor servicio de radio en todo el país.

\section{REFERENCIAS}

Ministerio de las TIC. (2018). Subdirección de Radiodifusión Sonora

https://www.mintic.gov.co/portal/inicio/Ministerio/ Direccion-de-Industria-de-

Comunicaciones/Subdireccion-deRadiodifusion-Sonora/

Ministerio de las TIC. (2019). Plan Técnico Nacional de Radiodifusión Sonora Frecuencia Modulada(F.M.). https://www.mintic.gov.co/portal/604/artic les62446_plan_tecnico_nacional_radiodifusi on_sonora_fm_actualizado_abril_2019.pd $\mathrm{f}$

Calvopiña H., W. L. (2016) Estudio de las afectaciones a la calidad del servicio de radiodifusión FM; problemas, causas y soluciones, caso de estudio en la ciudad de Riobamba. Pontificia Universidad Católica del Ecuador. Quito, Ecuador.

http://repositorio.puce.edu.ec/bitstream/handle/220 00/11288/informe\%20final\%20caso $\% 20 \mathrm{~d}$ e\%20estudio\%20William\%20Calvopina\% 2025-abr2016.pdf?sequence $=1 \&$ isAllowed $=y$

Molina, C. (2015). Transmisión de Señales. Factores que Afectan la Transmisión (p. 76).

https://silo.tips/downloadFile/transmisionde-seales

Gallardo, S. (2019). Elementos de sistemas de telecomunicaciones (2nd ed.). Madrid, España. 
Henze, A. (2018). Medición de Potencia en RF y Microondas

http://www2.electron.frba.utn.edu.ar/ jcecconi/Bib liografia/03\%20-

$\% 20$ Medicion $\% 20$ de $\% 20$ Potencia\%20en $\% 20 \mathrm{RF} \% 20 \mathrm{y} \% 20 \mathrm{micro}-$

ondas/Medicion\%20de\%20Potencia\%20$\% 20 U T N \% 202010 . p d f$

Ruiz S., A. O. (2018) Estudio de la cobertura de radiofrecuencia de la emisora Radio Libre 93.9 FM, de la ciudad de Babahoyo, Los Ríos. Universidad Técnica De Babahoyo, Ecuador.

http://dspace.utb.edu.ec/bitstream/handle/49000/47 60/-E-UTB-FAFI-ELECTRONICA000002.pdf? sequence $=1 \&$ is Allowed $=y$

Mejía, M., \& Pacheco, L. (2019). Medición de cobertura en potencia de la emisora Marina Stereo 90.7 F.M. en la localidad norte centro histórico de Barranquilla. Universidad Autónoma del Caribe. Colombia. 